\title{
SCALE EFFECTS AND CRACK PROPAGATION IN NON-LINEAR ELASTIC STRUCTURES
}

\author{
Y. W. MaI* and A. G. Atkins $\dagger$
}

Department of Mechanical Engineering, University of Michigan, Ann Arbor, Michigan 48104, U.S.A.

(Received 23 May 1975)

\begin{abstract}
Summary-New scaling laws are proposed for crack propagation in geometrically similar non-linear elastic structures. The stresses to cause cracking in large non-linear elastic structures are even smaller than those in larger linear structures.
\end{abstract}

\section{INTRODUCTION}

GURNEY AND HUNT'1 outlined the laws of similitude and crack propagation in linear elastic structures, where the load $(X)$ and the displacement $(u)$ relationship at constant crack area $(A)$ is specified by $(\partial u / \partial X)_{A}=u / X$. One of us has applied these laws to the particular problem of the scaling of ice-breaking forces as measured in towing tank experiments. ${ }^{2,3}$ Three scaling laws have been formulated, one relates to the stresses $(\sigma)$ at corresponding points of a model $(m)$ and a prototype $(p)$ cracked structure, and the other two to the fracture load and the corresponding fracture displacement. Thus,

$$
\frac{\sigma_{p}}{\sigma_{m}}=\frac{1}{\sqrt{\lambda}}, \frac{u_{p}}{u_{m}}=\sqrt{ } \lambda, \frac{X_{p}}{X_{m}}=\lambda^{z},
$$

where $\lambda$ is the linear scale factor of the prototype to the model. It has also been proved that under similar testing conditions, the prototype velocity $\left(\dot{a}_{p}\right)$ is always greater than the model velocity $\left(\dot{a}_{m}\right)$ and their relationship is given as

$$
\dot{a}_{p} / \dot{a}_{m}=\sqrt{ } \lambda
$$

There are, however, many practical situations in which the deformation of cracked structures, although reversible, is not proportional to the applied load. For such cases, the scaling laws given in equations (1) and

* Present address: Mechanical Engineering Department, Imperial College of Science and Technology, Exhibition Road, London SW7 2BX, England.

$\dagger$ Present address: Delta Materials Research Ltd., Box 22, Hadleigh Road, Ipswich, Suffolk, England.
(2) would not be applicable. It is the purpose of the present note, therefore, to develop new scaling laws which would be appropriate for describing crack propagation in geometrically similar non-linear elastic structures.

\section{ANALYSIS}

Consider a model cracked structure and a prototype cracked structure with a linear scale factor $(\lambda)$. Let their non-linear load-displacement relationship at constant crack area be

$$
X=k(A) u^{n},
$$

where $k(A)$ is any mathematical function in $A$ and $n$ is the degree of non-linearity. From the concepts of non-linear elastic fracture mechanics introduced by Gurmey and Ngan, ${ }^{4}$ we have

$$
R=\left(\frac{\partial \Omega}{\partial A}\right)_{X}=\left(\frac{\partial(X u-\Lambda)}{\partial A}\right)_{X},
$$

where $\Omega$ and $\Lambda$ are the complementary and strain energy functions, and $R$ is the fracture toughness. Thus,

$$
R=-\frac{k^{\prime}}{(n+1)} k^{-(n+1) / n} X^{(n+1) / n},
$$

where $k^{\prime}=\mathrm{d} k / \mathrm{d} A$, the prime notation is used for the area derivative. By applying equation (5) to the model and prototype cracked structures, we obtain

$$
\frac{R_{p}}{R_{m}}=\frac{k_{p}^{\prime}}{k_{m}^{\prime}}\left(\frac{k_{m}}{k_{p}}\right)^{(n+1) / n}\left(\frac{X_{p}}{X_{m}}\right)^{(n+1) / n}
$$

Equation (3) gives

and

$$
\frac{k_{p}}{k_{m}}=\left(\frac{X_{p}}{X_{m}}\right)\left(\frac{u_{m}}{u_{p}}\right)^{n}
$$

$$
\frac{k_{p}^{\prime}}{\overline{k_{m}^{\prime}}}=\frac{\left(\mathrm{d} / \mathrm{d} A_{p}\right)\left(k_{p}\right)}{\left(\mathrm{d} / \mathrm{d} A_{m}\right)\left(k_{m}\right)}=\frac{1}{\lambda^{2}}\left(\frac{X_{p}}{\bar{X}_{m}}\right)\left(\frac{u_{m}}{u_{p}}\right)^{n} .
$$



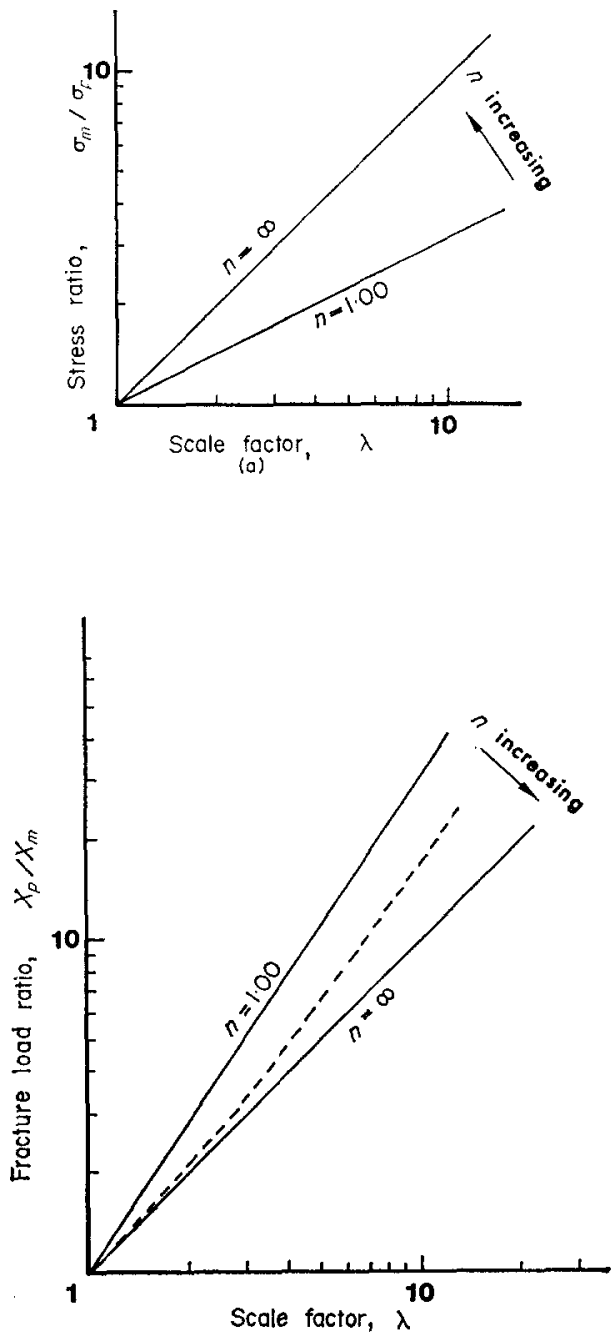

(b)

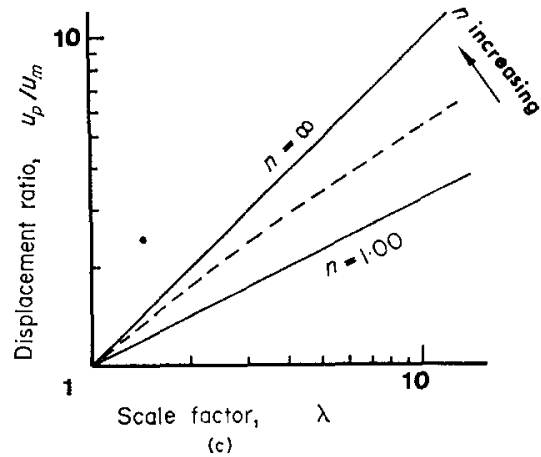

FIg. 1. (a) Variations of $\sigma_{m} / \sigma_{p}$ with $\lambda$. (b) Fracture load ratios $X_{p} / X_{m}$ vs $\lambda$. (c) Fracture displacement ratios $u_{p} / u_{m}$ vs $\lambda$.
Combining equations (6) and (7), this gives

$$
\frac{R_{p}}{R_{m}}=\frac{1}{\lambda^{2}}\left(\frac{X_{p}}{X_{m}}\right)\left(\frac{u_{p}}{u_{m}}\right) .
$$

Now consider stresses and strains at corresponding points in the model and prototype, where

$$
\left.\begin{array}{ll}
X_{p}=\beta \sigma_{p} \lambda^{2}, & X_{m}=\beta \sigma_{m}, \\
u_{p}=\alpha \lambda \sigma_{p}^{1 / n}, & u_{m}=\alpha \sigma_{m}^{1 / n},
\end{array}\right\}
$$

$\alpha$ and $\beta$ in the above equation are some constants.

Therefore, using equation (8) in conjunction with expressions in equation (9), wo could derive the scaling laws for non-linear elastic structures as

$$
\left.\begin{array}{rl}
\frac{R_{p}}{R_{m}} & =\left(\frac{u_{p}}{u_{m}}\right)^{n+1} \frac{1}{\lambda^{n}}, \\
\frac{R_{p}}{R_{m}} & =\lambda\left(\frac{\sigma_{p}}{\sigma_{m}}\right)^{(n+1) / n}, \\
\frac{R_{p}}{R_{m}} & =\lambda^{-(n+2) / n}\left(\frac{X_{p}}{X_{m}}\right)^{(n+1) / n}
\end{array}\right\}
$$

For situations where the model and prototype have invariant fracture toughness so that $R_{p}=R_{m}$, equation (10) can be simplified to

$$
\left.\begin{array}{rl}
\frac{u_{p}}{u_{m}} & =\lambda^{n /(n+1)}, \\
\frac{\sigma_{p}}{\sigma_{m}} & =\lambda^{-n /(n+1)}, \\
\frac{X_{p}}{X_{m}} & =\lambda^{(n+2) /(n+1)} .
\end{array}\right\}
$$

It should be noted that the new scaling laws thus derived depend explicitly on the degree of non-linearity of the elastic structures. When $n=1$, i.e. structures become linear elastic, equation (11) degenerates to those shown in equation (1) as expected; and when $n$ is large, i.e. structures become highly non-linear, both the fracture load and displacement vary linearly, and the stress varies inversely with the scale factor. Figs. 1(a)(c) show the variations of $\sigma_{m} / \sigma_{p}, X_{p} / X_{m}$ and $u_{p} / u_{m}$ with $\lambda$ for varying degree of non-linearity $(n)$.

\section{DISCUSSION}

A striking conclusion follows from the second of equation (11), namely, that the stresses to cause cracking in large non-linear structures are even smaller than those in large linear elastic structures, i.e. $\sigma_{p}=\sigma_{m} / \lambda$ as opposed to $\sigma_{p}=\sigma_{m} / \sqrt{ } \lambda$. This observation does not seem to have been presented previously. The difference in scaling also suggests that non-linear laboratory test-pieces should display generalized yielding more readily than linear test-pieces.

It should also be noted that in the derivation of equation (10) or (11) for scaling effects in nonlinear elastic cracked structures, we assume geometrical similarity between the model and prototype both under loaded and unloaded conditions. 
Whether this assumption is realistic or not has to be experimentally determined but unfortunately there is practically no published information on the subject for us to do any evaluation of the analysis. In some preliminary studies, however, $\mathrm{Ngan}^{5}$ has shown that for splitting of slender beams by shear forces (see also ref. (4)), the degree of nonlinearity decreases slightly as $\lambda$ increases, which indicates that the deformed geometries of the model and prototype may have been slightly dissimilar under loaded conditions. The results for the variation of $X_{p} / X_{m}$ (according to $\mathrm{Ngan}^{5}$ ) and $u_{p} / u_{m}$ vs $\lambda$ are shown in dotted lines in Figs. 1(b) and (c).

Consider now scale effects on crack velocities in non-linear elastic structures. For quasi-static cracking ${ }^{1,4}$ in reversible elastic structures to occur, Gurney and Hunt ${ }^{1}$ have shown that

$$
X \mathrm{~d} u=t R \mathrm{~d} a+\mathrm{d} \Lambda,
$$

where $t$ is test-piece thickness, $a$ the crack length and $\Lambda$, strain energy function for the non-linear elastic structures with load-displacement relationships defined by equation (3), is given by

$$
\Lambda=\frac{X u}{n+1} \text {. }
$$

By assuming $R$ to be constant during the whole fracture process, equations (12) and (13) together yield the ratio of the crack velocity to the driving velocity, i.e. crosshead speed of test machine $(\dot{u})$, as

$$
\frac{\dot{a}}{\dot{u}}=\frac{n X}{R t(n+1)+u \mathrm{~d} X / \mathrm{d} a} .
$$

From equation (12), we have

$$
R=-\frac{1}{t}\left(\frac{\partial \Lambda}{\partial a}\right)_{u}=\frac{-k^{\prime}}{n+1}\left(\frac{X}{k}\right)^{(n+1) / n}
$$

and differentiating it with respect to crack length $a$ and in conjunction with equation (14), we obtain

$$
\frac{\dot{a}}{\dot{u}}=-\frac{k^{\prime}}{k^{\prime \prime}} \frac{n+1}{u t} \text {. }
$$

When the crosshead speeds or driving velocities, $\dot{u}_{p}$ and $\dot{u}_{m}$ are assumed equal, the ratio of the corresponding crack velocities in the prototype and model is

$$
\frac{\dot{a}_{p}}{\dot{a}_{m}}=\frac{k_{p}^{\prime}}{k_{m}^{\prime}} \frac{k_{m}^{\prime \prime}}{k_{p}^{\prime \prime}} \frac{t_{m}}{t_{p}} \frac{u_{m}}{u_{p}}
$$

Since $t_{m} / t_{p}=\lambda^{-1}, u_{p} / u_{m}=\lambda^{n /(n+1)}$ (from the first of equation (11)) and $\left(k_{p}^{\prime} / k_{m}^{\prime}\right)\left(k_{m}^{\prime \prime} / k_{p}^{\prime \prime}\right)=\lambda^{2}$, equation (17) is reduced to

$$
\dot{a}_{p} / \dot{a}_{m}=\lambda^{1 /(n+1)} \text {. }
$$

It can be seen that, when $n=1$ for the linear elastic case, $\dot{a}_{p} / \dot{a}_{m}=\sqrt{ } \lambda$, which is the same as that given in equation (2).

We suggest that experimental effort should be devoted to confirm these non-linear scaling laws, particularly when they suggest such dangerous possibilities as easier cracking in large non-linear structures at lower stresses than cracking in large linear structures.

\section{REFERENCES}

1. C. Gurney and J. Hunt, Proc. Roy. Soc. A299, 508 (1967).

2. A. G. Atkins and R. M. Caddell, Int. J. mech. Sci. 16, 541 (1974).

3. A. G. Atkins, J. Ship Res. 19, 40 (1975).

4. C. Gurney and K. M. Ngan, Proc. Roy. Soc. A325, 207 (1971).

5. K. M. NGan, Ph.D. Thesis, University of Hong Kong (1971). 